\title{
Métodos para el Estudio de la Estabilidad de Voltaje en Sistemas de Potencia
}

\author{
John E. Candelo, Gladys Caicedo y Ferley Castro \\ Univ. del Valle, Esc. de Ingeniería Eléctrica y Electrónica, Grupo de Investigación en Alta Tensión, \\ Ciudad Universitaria Meléndez, Calle 13 № 100-00, Cali-Colombia \\ (e-mail: glacadel@univalle.edu.co)
}

\section{Resumen}

Este artículo presenta una revisión crítica actualizada de los métodos de estudio de la estabilidad de voltaje en sistemas de potencia. Se realiza la descripción y clasificación en orden cronológico de cada una de las técnicas de estudio, para la detección y predicción de la estabilidad de voltaje desde la identificación del problema en los años 60's. También se presentan las modificaciones a las técnicas originales y la utilización en la solución a problemas comunes de estabilidad de voltaje presentados en los sistemas de potencia. La comparación de las técnicas se realiza de acuerdo a la información de la literatura y se discuten las ventajas y desventajas de cada técnica de estudio en la solución de los problemas de estabilidad de voltaje.

Palabras clave: estabilidad de voltaje, sistemas de potencia, detección de estabilidad, revisión de la literatura

\section{Methods for studying Voltage Stability in Power Systems}

\begin{abstract}
This paper presents the state of the art of methods for studying voltage stability in power systems. Description and chronological classification of each technique for detection and prediction of voltage stability, since the identification of the problem in the 60's, are done. Also, modifications to the original techniques and their utilization to solve common problems of voltage-stability in the power system are presented. Comparisons of the different techniques were done according to literature information and the advantages and disadvantages of each technique in the solution of voltage stability problems are discussed.
\end{abstract}

Keywords: voltage stability, power systems, stability detection, literature review 


\section{INTRODUCCIÓN}

La estabilidad de voltaje ha sido desde los años 60's un tema de gran importancia debido a los colapsos de voltaje presentados mundialmente (Kundur, 1994; Taylor, 1994; Bucciero y Terbrueggen, 1998; Kundur et al., 2004). Todos estos eventos dieron originen a investigaciones y propuestas de métodos de estudio para detectar y predecir la inestabilidad con buena precisión. La realización de estos estudios requiere de gran cantidad de datos de la configuración, modelos y parámetros del sistema de potencia, con los cuales se desarrollan técnicas computacionales adecuadas; sus resultados muestran los estados del sistema, márgenes y límites de operación, con los cuales se definen las acciones de prevención y corrección del problema. Varias técnicas de estudio han sido desarrolladas y algunas de ellas clasificadas en varios artículos (Kundur, 1994; Taylor, 1994; Ajjarapu y Lee, 1998; Grigsby, 2001; Chowdhury y Taylor, 2000; Sanaye-Pasand y Rezaei-Zare, 2003), pero no se cubre en forma general el tema, no se hace referencia a desarrollos importantes, no se ordenan en orden cronológico y no se presentan desarrollos recientes; esto hace difícil la identificación y selección de una técnica adecuada de estudio.

Este artículo presenta el estado del arte de los métodos de estudio de la estabilidad de voltaje clasificado en orden cronológico; se presenta la evolución de sus técnicas y la comparación de los métodos, para mostrar las ventajas y desventajas de la implementación en sistemas de potencia.

\section{MÉTODOS DE ESTUDIO DE LA ESTABILIDAD DE VOLTAJE}

Los métodos de estudio se basan en técnicas de detección y predicción de la estabilidad de voltaje. La detección se utilizan para obtener el estado de operación de los nodos y la predicción encuentran los márgenes y límites de voltaje para la operación segura de los nodos. Estos estudios se realizan por medio de métodos analíticos y de monitoreo.

\section{Métodos Analíticos}

Se utilizan para estudiar en detalle las causas y los efectos de la estabilidad de voltaje, así como el comportamiento de las variables y los elementos del sistema. Los resultados de estos estudios se utilizan para definir soluciones preventivas y correctivas de la inestabilidad de voltaje. Cada uno de estos métodos utiliza técnicas matemáticas que requieren de herramientas computacionales eficientes para el análisis en grandes sistemas de potencia. Los estudios analíticos se pueden realizar en forma estática, como los métodos basados en flujos de carga convencionales y los flujos de carga progresivos. También se pueden realizar análisis dinámicos de los estados del sistema como los transitorios, de pequeña señal y casi estacionarios.

Flujos de potencia convencionales

Son flujos de potencia estáticos que representan la variación de los voltajes en los nodos del sistema con respecto al cambio de la potencia de la carga. Estas técnicas calculan los estados, límites y márgenes de estabilidad de voltaje en el sistema de potencia, para el estado normal de operación y después de contingencias. Sus resultados se utilizan para graficar el voltaje versus la potencia y calcular los límites, márgenes, índices de proximidad a la inestabilidad e identificación de las áreas, nodos o elementos débiles del sistema. Las técnicas desarrolladas para los métodos de flujos convencionales son: análisis de sensibilidad, análisis por equivalentes de red, singularidad de la matriz Jacobiana, diferencia vectorial y técnicas basadas en energía.

1) Análisis de sensibilidad: Esta técnica se basa en el análisis de la variación del voltaje con respecto a la potencia, para determinar los márgenes y límites de estabilidad de voltaje, así como las áreas y zonas más sensibles del sistema. En 1983 se analizó la sensibilidad del voltaje con respecto a la potencia reactiva de un nodo de carga, dV/dQ (Berntsen et al., 1983). En 1990 se midió la distancia al colapso con matrices de sensibilidad de voltaje en los nodos y la variación de reactivos de la carga hasta el límite $\Delta$ Qi (Flatabo et al., 1990). En 1991 se analizó la sensibilidad del voltaje ante el cambio de potencia activa $\mathrm{dV} / \mathrm{dP}$ y la potencia reactiva $\mathrm{dV} / \mathrm{dQ}$, y se definió un índice de proximidad al colapso de voltaje para cada nodo (Hawkins et al., 1991). En este mismo se utilizó la sensibilidad 
$\mathrm{dV} / \mathrm{dQ}$ para obtener un índice de proximidad al límite, el peor escenario de operación del sistema en estado estable WVII y la probabilidad de que este ocurra PWVII. En 1992 se utilizó la sensibilidad para determinar las reservas de reactivos y controlar la estabilidad con compensación en los nodos críticos (Begovic y Phadke, 1992). En 1999 se seleccionaron y clasificaron las contingencias, basados en un índice entrega de reactivos desde el punto normal de operación hasta un punto después de una contingencia, llamado índice de soporte de reactivos (RSI) y un método de filtrado para seleccionar las contingencias más criticas (Vaahedi et al., 1999a). También en este año se evaluaron las herramientas de planeación de vares y las de flujo óptimo, para la inclusión de las restricciones de estabilidad de voltaje y de niveles de voltaje en grandes sistemas de potencia (Vaahedi et al., 1999b). En el año 2002 se analizó la sensibilidad de los reactivos del nodo generador ante cambios de carga, para determinar la estabilidad relativa del sistema, el grupo de generadores que causan la inestabilidad de voltaje y los nodos de carga que causan colapsos de voltaje en grandes sistemas de potencia (Aumuller y Saha, 2002). En el 2005 se utilizó la sensibilidad para analizar la estabilidad después de contingencias, combinada con sensibilidades lineales y análisis de vectores propios para la clasificación de las contingencias (Amjady y Esmaili, 2005). También en este año se utilizó la sensibilidad para determinar las posibles contingencias y las áreas que se inestabilizan después de cada evento (Capitanescu y Van Cutsem, 2005). En el 2006 se utilizaron para calcular la cargabilidad y el margen de seguridad de voltaje inicial, complementados con técnicas de optimización del flujo de potencia para el ajuste de los pasos de carga cerca al límite de estabilidad (Zarate y Castro, 2006); este método se puede implementar utilizando programación no lineal (Zarate et al., 2006).

2) Reducción de la matriz Jacobiana: Se basa en la reducción de la matriz Jacobiana para encontrar las expresiones que definan los límites de estabilidad, los márgenes del sistema, la proximidad al colapso de voltaje y los nodos y elementos con deficiencia de reactivos en la red. Estas técnicas se dividen en: singularidad de la matriz Jacobiana y análisis modal.

a. Singularidad de la matriz Jacobiana: Se basa en la reducción de la matriz Jacobiana del flujo de potencia con el fin de encontrar la singularidad, la cual define el límite de estabilidad de voltaje de los nodos. En 1975 se utilizó la singularidad de la matriz Jacobiana para determinar el punto de colapso del sistema (Venikov et al., 1975). En 1989 se describió el colapso de voltaje como un problema de bifurcaciones y utilizando la técnica de la singularidad de la matriz se calculó la proximidad al límite de estabilidad; el límite se define como el punto dónde se encuentra una bifurcación en la curva de estabilidad de voltaje (Thomas y Chiang, 1989). En 1992, una nueva técnica de singularidad, reduce la matriz Jacobiana a pocas variables, con el fin de calcular rápidamente índices de estabilidad para aplicaciones en línea (Lof et al., 1992). En 1993, se definió un índice de proximidad al colapso de voltaje como la mínima singularidad de la matriz Jacobiana del flujo de potencia, encontrando los vectores propios, izquierdo y derecho de la matriz; la mínima singularidad de la matriz define la proximidad al límite de estabilidad, el vector singular derecho representa el índice de sensibilidad de los voltajes y ángulos de los nodos, y el vector izquierdo la dirección de mayor sensibilidad al cambio de potencia activa y reactiva (Lof et al., 1993). En 1998 se propone un método para calcular la proximidad al colapso de voltaje basado en flujos de carga operacionales y la sensibilidad de variables de control de reactivos, utilizando un indicador de la distancia desde el estado actual hasta el límite de estabilidad llamado L-Index (Thukaram et al., 1998). En el año 2006 se comparan diferentes formulaciones de los índices de estabilidad de voltaje derivados de la matriz, en presencia de la regulación de voltaje secundario, para ser utilizado en el cálculo del máximo valor singular en la función objetivo de un flujo óptimo de potencia reactiva (Berizzi et al., 2006). En este mismo año se utilizó el método de valores singulares de la matriz Jacobiana para calcular la dirección del incremento de las cargas basadas en el manejo de eigenvectores (Bedoya y Castro, 2006). En el año 2006 se presentó un método para encontrar el punto máximo de carga por medio de la evaluación de las posibles direcciones que toma la generación utilizando análisis de singularidad, representando la relación entre la dirección de la generación y la máxima carga (Sode-Yome et al., 2006).

b. El análisis Modal: Se basa en la reducción de la matriz Jacobiana con el fin de encontrar los valores y vectores propios de estado. Es un método indirecto del cálculo de la sensibilidad dV/dQ. En 1992 se utilizó para determinar la estabilidad de voltaje ante variaciones de carga y la participación de reactivos en los nodos, generadores y líneas (Gao et al., 1992). En 1995, se utilizaron valores 
propios de la matriz de admitancia para establecer la seguridad en un sistema de potencia multimáquinas; se utilizan los índice de estabilidad de voltaje VSI y control de voltaje VCI (Vournas, 1995). En 1996 se utilizó el análisis modal en un sistema de transmisión DC, el cual permitía ajustar la estructura de los lazos de un sistema de control, con el fin de ubicar los ceros críticos de la matriz (Aik y Andersson, 1997) y en 1998 se extendió este trabajo para un convertidor triple (Aik y Andersson, 1998). En el año 2000 se utilizó para el análisis y la clasificación de continencias críticas, permitiendo definir los nodos críticos y ubicar los elementos de control (Hae-Kon et al., 2000). En 2003 se utilizaron para estudiar la seguridad del sistema con FACTS (El-Sheikhi et al., 2003). En este mismo año se utilizaron factores de participación modal para clasificar la contribución de cada generador del sistema a la seguridad (Huang et al., 2003). En el año 2007 se utilizó el análisis modal, el flujo de potencia, y las curvas PV y QV para asistir a los operarios en la configuración y el análisis de la estabilidad de voltaje en un punto de operación (Sharma y Ganness, 2007).

3) Equivalente de red: Se basa en obtener los equivalentes del sistema para compararlos con los de la carga de un nodo. En 1992 se definió un índice como la impedancia de red dividida en la impedancia de carga (Chebbo et al., 1992). En 1997 se utilizó esta técnica para obtener las curvas PV y definir un índice proximidad al colapso de voltaje en MW (Nagao et al., 1997). En el 2001 se construyeron curvas activa y reactiva $P Q$, con el fin de estimar el margen de potencia reactiva (Sobierajski et al., 2001). En el 2002 se calcularon los límites y márgenes de estabilidad de voltaje y se definió la potencia máxima de transferencia por medio de curvas P-Q (Haque, 2002). En el año 2003 se utilizaron para definir regiones de operación de potencia reactiva y activa, basados en planos PQ (Haque, 2003a; 2003b). En el año 2004 se utilizaron equivalentes de impedancia para estudiar la estabilidad de voltaje con regiones P-Q; se utiliza distribución rectangular probabilística de la carga para estimar la probabilidad de la violación del voltaje crítico en nodos con carga distribuida uniformemente (Sobierajski y Fulczyk, 2004). En el 2007 se calcula el margen de voltaje con la potencia aparente y el equivalente de admitancia en un punto (dS/dY), para determinar el punto de inicio de la eyección de carga (Wiszniewski, 2007).

4) Técnicas basadas en fasores y vectores de voltaje: Se basan en la diferencia fasorial de las variables asociadas a la estabilidad de voltaje, la cuales se determinan mediante flujos de potencia en estado normal y crítico. En 1988 se definió un índice basado en la diferencia angular de los voltajes entre dos nodos y un índice de proximidad a la inestabilidad de voltaje VIPI, el cual calculaba la diferencia de ángulos entre dos nodos (Tamura et al., 1988). También se ha definido el índice de proximidad a la inestabilidad de voltaje VIPIt para la prevención y el control de emergencias (Esaka et al., 2004). En el 2004 se presentó el índice de margen de estabilidad de voltaje (VSMI), basado en las diferencias angulares máximas de los voltajes y la del estado normal de operación (He et al., 2004). En el 2006 se propone el índice de proximidad a la inestabilidad de voltaje VMPI, definido por diferencia vectorial entre el voltaje de operación y límite inferior del voltaje (Kataoka et al., 2006).

6) Técnicas basadas en energía: Se basan en la diferencia entre las soluciones de un flujo de potencia después de un disturbio, llamado solución de alto voltaje y la solución de un flujo de potencia en el mínimo punto de inestabilidad llamado solución de bajo voltaje; esto define la máxima transferencia de potencia, el límite y margen de estabilidad de voltaje. En 1990, se presentó una técnica basada en energía de dos sistemas, para medir la proximidad al colapso de voltaje (DeMarco y Overbye, 1990). En 1993 se propuso la aplicación en línea de estas técnicas, para brindar información de las condiciones del sistema que permitían realizar las operaciones de control (Overbye, 1993).

Flujos progresivos

Son métodos de análisis estáticos basados en flujos de potencia continuos que buscan con precisión los límites de estabilidad de voltaje; estos métodos pertenecen a una clase general de ecuaciones algebraicas no lineales conocidos como métodos path-following (Kundur, 1994). En 1989 se presentó un algoritmo que obtenía el límite de estabilidad con gran precisión numérica (Ajjarapu y Christy, 1989). En 1991 se presentó el flujo de potencia continuo, el cual utilizaba un vector tangente de predicción y un algoritmo de corrección para encontrar el límite (Ajjarapu y Christy, 1991). En 1993 se utilizó este método para el análisis en grandes sistemas de transmisión AC/DC (Cañizares y 
Alvarado, 1993). En 1997 se mejoró la velocidad de cálculo para aplicaciones en línea (De Souza et al., 1997). En este mismo año se utilizó una técnica de continuación para calcular un índice con el voltaje actual y el de máxima cargabilidad, antes y después de cada continencia; se dibujaron curvas PQ-V para representar la variación del voltaje ante el aumento de la potencia activa y reactiva de la carga (Hsiao-Dong Chiang et al., 1997). En 1998 se utilizaron para encontrar el límite de estabilidad después de contingencias y definir estrategias de mínima eyección de carga (Feng et al., 1998). En el año 2000 se utilizaron para definir el índice de incremento de carga LII, basado en encontrar la máxima cargabilidad de un nodo (Yang et al., 2000). En el 2001 se presentó un método para estimar el margen de flujo de potencia de las líneas de transmisión entre dos regiones, el cual a partir de las curvas de variación del voltaje con respecto al flujo de potencia activa de las líneas de transmisión, calculaba el mínimo margen de operación estable ante contingencias criticas (Song et al., 2001). En el año 2004 se propuso un método de análisis de la estabilidad de voltaje para grandes sistemas de potencia basado en el flujo de potencia continuo híbrido, el cual permite graficar rápidamente las curvas PV en un nodo; utilizando un método de predicción no lineal se grafica la parte superior de la curva hasta los puntos cercanos al límite y un método de predicción lineal para la parte inferior (Mori y Kojima, 2004). También en este año se utilizó este método para el análisis de los límites de potencia reactiva entregada por los generadores que regulan voltaje (Yorino et al., 2004). En el 2005 se presentó un algoritmo de continuación de flujo de potencia trifásico en coordenadas polares, para el análisis de estabilidad de voltaje en sistemas trifásicos desbalanceados (Zhang et al., 2005). En el 2006 se utilizó una técnica de continuación para definir los parámetros de carga utilizados en análisis dinámicos casi estacionarios (Wang et al., 2006). En este mismo año se incluye en la técnica de continuación, los parámetros de pérdidas activas de las ramas del sistema de transmisión (Nino et al., 2006). También se analizó la estabilidad de voltaje dinámica, utilizando una técnica de continuación para trazar las rutas de equilibrio del sistema y luego se analizó cada punto de equilibrio con factores de participación modales (Su et al., 2006). En el año 2007 se probó esta técnica con modelos mejorados de cargas y generadores (Lopez-Luis et al., 2007). En este mismo se utilizaron expansiones de Taylor y Perturbación para formar la matriz Jacobiana sin reducción y la técnica de continuación para trazar los puntos de equilibrio del sistema, con el fin de realizar un análisis dinámico de la estabilidad de voltaje (JiaKuan y Xin, 2007).

Técnicas de análisis dinámico

Estas técnicas se basan en soluciones de ecuaciones algebraicas en el dominio del tiempo (Kundur, 1994; Taylor, 1994) y se utilizan para el análisis de transitorios y estabilidad de pequeña señal. Estas técnicas permiten crear diferentes escenarios que incluye el estado de operación normal y contingencias, y se utilizan para determinar los tiempos de respuestas y comportamientos de los elementos ante los diferentes eventos. Varias simulaciones en el tiempo fueron realizadas a finales de los años 70s y principios de los 80s para representar el fenómeno de inestabilidad de mediano tiempo (Gelopulos, 1979) y largo tiempo (Schulz y Turner, 1980). En los años 90s se utilizaron para analizar colapsos de voltaje en sistemas AC/DC, con la función de Lyapunov evaluada en un instante de tiempo (Cañizares, 1991). También para la clasificación de contingencias y reducción del tiempo de análisis de la estabilidad de voltaje (Jasmon y Lee, 1993). Se utilizaron para estudiar el comportamiento del colapso de voltaje con cargas dinámicas (Xu y Mansour, 1994). En 1994 se propuso el análisis casi estacionario para la estabilidad dinámica de voltaje del sistema, el cual consistía en modificar las ecuaciones transitorias dinámicas por relaciones de equilibrio; esta herramienta es muy eficiente en el tiempo, pero no representa totalmente la estabilidad transitoria (Van Cutsen et al., 1994; Van Cutsen et al., 1995). La comparación con otras técnicas de simulación dinámica se realizó en un sistema de prueba (Van Cutsem y Vournas, 1996) y luego en el sistema real Hydro-Quebec (Van Cutsem y Mailhot, 1997). A finales de los 90s se evalúan los comportamientos dinámicos del sistema ante diferentes contingencias y se entrenan redes neuronales para la identificación del colapso (Schmidt, 1997). En 1998 se analizó la estabilidad de voltaje transitoria después de disturbios con el método cuadrático de Lyapunov, para sistemas de potencia pequeños (Halim et al., 1998a) y sistemas grandes con disturbios en la red (Halim et al., 1998b). En el 2001 se analizó el impacto de las fuentes dinámicas de reactivos sobre la estabilidad de voltaje, ante diferentes disturbios del sistema (Garng y Zhang, 2001). En el año 2006 se propuso un índice del margen de estabilidad de voltaje transitorio, basado en la aproximación cuadrática de los límites de la región de estabilidad, para estimar el tiempo de despeje crítico del transitorio de voltaje y para clasificar las contingencias (Yihong Wang et al., 2006) En el 2006 se utilizaron 
simulaciones dinámicas por el método casi estacionario para aproximar la estabilidad de voltaje de tiempo largo (Wang et al., 2006). En el 2006 se estudió la estabilidad de pequeños disturbios con simulaciones de estado casi estacionario, utilizando modelos estáticos de carga y TAPs bajo carga (An Ning, 2006). En el 2007 se utilizaron métodos dinámicos para encontrar la mejor ubicación y requerimientos mínimos de la eyección de carga (Koessler et al., 2007).

\section{Monitoreo}

Son técnicas basadas en la toma de datos reales que permiten definir estados de operación, zonas críticas de la red, límites y márgenes de estabilidad de voltaje; ellos se utilizan como herramienta para la detección y predicción de la estabilidad de voltaje en línea o fuera de línea. En 1990 se midieron fasores de voltaje para analizar la estabilidad de voltaje en tiempo real, para el control secundario de voltaje, estimar el estado del sistema y calcular los márgenes de estabilidad de voltaje (Mili et al., 1990). En 1993 se estimó la estabilidad utilizando el monitoreo con redes neuronales artificiales (Mori y Tamaru, 1993). En 1997 se propuso una técnica de monitoreo de la seguridad de voltaje del sistema por medio de redes neuronales y lógica difusa (Chih-Wen et al., 1998). En 1998 se presentó un método de predicción y monitoreo en cada nodo del sistema, basado en redes neuronales entrenadas con magnitudes de voltaje y ángulos de fase (Belhadj et al., 1998). En 1999 se calculó la impedancia equivalente Thevenin con mediciones de voltaje, corriente y el valor la demanda de carga en el nodo, para predecir la cercanía a la inestabilidad VIP y accionar la eyección de carga (Vu et al., 1999). En el año 2000 se utilizaron mediciones de voltaje y corriente para definir la proximidad al límite de estabilidad basada en la metodología VIP, por medio de la diferencia entre la máxima potencia transmitida estimada y la potencia medida (Julian et al., 2000). También en este año se presentó una metodología para determinar la estabilidad de voltaje en línea, modelando el estado de operación del sistema con la ayuda de un estimador de estado y calculando el punto de colapso a través de técnicas de extrapolación basadas en el comportamiento de vectores tangentes (De Souza et al., 2000). En el año 2001 se presentó una técnica basada en la medición y tabulación de variables del sistema, llamada modelo "Black-Box", la cual se utiliza para evaluar la estabilidad de voltaje ante grandes disturbios (Sami Repo, 2001). En el 2003 se desarrolló una técnica para predecir el margen de estabilidad con equivalentes Thevenin del sistema, a partir de mediciones de voltajes y corrientes, históricas y actuales (Soliman et al., 2003). En el año 2003 se midieron fasores de voltaje para identificar nodos, segmentos o tramos críticos de estabilidad de voltaje y definir la ubicación de FACTS (Sharma et al., 2003). En el 2003 se calculó el límite y el margen de estabilidad de voltaje con voltajes locales, potencia activa y reactiva medidos de la red (Haque, 2003a; 2003b). En el 2003 se monitoreó la estabilidad de voltaje con las reservas de reactivos de los principales generadores de la red (Lixin Bao et al., 2003). En el 2004 se determinó el equivalente Thevenin del sistema y los márgenes de estabilidad de voltaje, utilizando PLCs, y medidores de voltajes y corrientes en subestaciones (Santos y Barbosa, 2004). En el 2004 se presentó la detección y predicción de la estabilidad de voltaje con medidores de fasores de voltaje y corriente PMUs y un GPS, calculando la diferencia de ángulos de los fasores en diferentes subestaciones (Uhlen et al., 2004). También en este mismo año se utilizaron curvas de medición de voltaje y corriente V-I, para estimar la carga crítica de colapso (Haque, 2004). Se monitorearon contingencias y la estabilidad de voltaje, con redes neuronales entrenadas por mediciones de potencia activa y reactiva (Chakrabarti y Jeyasurya, 2004). En el 2006 se evaluó la estabilidad de voltaje con redes neuronales feed-forward entrenadas con el algoritmo para el cálculo de un índice L-Index (Kamalasadan et al., 2006). Se analizó la seguridad de la estabilidad de voltaje con medición de fasores sincronizados, para estimar los modelos del sistema y de la carga; se calculan con los datos las curvas PV y los límites de estabilidad (Parniani et al., 2006). En el año 2007 se presentó un control preventivo y de monitoreo de la estabilidad de voltaje en línea con datos medidos de la red, para estimar las direcciones que toma la carga y estimar los límites de estabilidad (Zhao et al., 2007).

\section{COMPARACIÓN DE LAS TÉCNICAS}

Varias comparaciones han sido realizadas para determinar la precisión, eficiencia, aplicabilidad, y otras características de los métodos de estudio y sus técnicas de estabilidad de voltaje que muestran las ventajas y desventajas en aplicaciones comunes de solución. En 1993 se compararon análisis estáticos y dinámicos en un sistema de potencia pequeño (Morison et al., 1993). En 1996 se 
compararon índices de reducción de la matriz jacobiana, valores singulares y vectores propios (Cañizares et al., 1996). En 1997 se simularon técnicas estáticas y dinámicas para la estabilidad de voltaje (Nagao et al., 1997). En el 2000 se compararon los índices "L-Index", diagonal de la matriz Jacobiana "Id", cambio ángulos de voltaje fase con respecto a la potencia activa y reactiva, sensibilidad del ángulo del voltaje con respecto a la potencia activa y sensibilidad de voltaje con respecto a la potencia reactiva (Sinha y Hazarika, 2000). En este mismo año se comparan los análisis dinámicos y las curvas de reactivos versus voltaje, QV (Chowdhury y Taylor, 2000). En el año 2003 se muestra un resumen de las técnicas prácticas de la estabilidad y sus ventajas (IEEE/PES Power System Stability Subcommittee, 2003). En el 2005 se verificó la precisión que tienen los índices estáticos en la representación de la estabilidad de voltaje, utilizando análisis de pequeña señal y dinámicos en el dominio del tiempo (Huadong et al., 2005). También se probó la efectividad de los índices de estabilidad para predecir la severidad de las contingencias (Limbu et al., 2005). En el 2006 se analizaron y se compararon índices de sensibilidad, singulares, modales, estabilidad de línea y de voltaje en los nodos, para diferentes incrementos de carga (Reis y Barbosa, 2006). En el 2006 se evaluaron varios índices de predicción del colapso de voltaje dinámico, tales como: proximidad al colapso de voltaje, líneas de transmisión y de margen de potencia, y estabilidad de transferencia de potencia (Muhammad Nizam et al., 2006). En este mismo año se hace una comparación de varios índices derivados de la singularidad de la matriz Jacobiana (Berizzi et al., 2006).

La Tabla 1 muestra la comparación de los métodos de estudio estático y dinámico, por medio de índices que definen la importancia que tienen en aplicaciones reales, para la prevención y corrección de la estabilidad de voltaje; estos índices se escogieron de acuerdo a las evaluaciones expuestas por los autores y la importancia que tienen en la implementación en sistemas de potencia. El primer índice corresponde a la evaluación de los métodos de estudio en grandes sistemas de potencia, considerando tiempos de implementación, tiempos de ejecución, precisión en los resultados y consumo de recursos computacionales. El segundo índice muestra la eficiencia, precisión y aplicabilidad de los métodos de estudio en línea con el sistema. El tercer índice ilustra la flexibilidad que tienen los métodos para ajustarse a constantes cambios producidos en el sistema. El cuarto índice muestra la evaluación del menor tiempo de ejecución de los métodos. El quinto índice evalúa la precisión que ofrece cada método para determinar los límites y márgenes de estabilidad de voltaje. El sexto índice evalúa las menores limitaciones de implementación que tiene cada método para el estudio e integración con las aplicaciones comunes de análisis. El séptimo índice evalúa los menores costos para la implementación de los métodos (software y hardware).

Tabla 1: Comparación de Métodos de Estudio de la Estabilidad de Voltaje

\begin{tabular}{|l|c|c|c|c|c|c|c|}
\hline & \multicolumn{7}{|c|}{ Índices de Evaluación } \\
\cline { 2 - 8 } Métodos de Estudio & $\begin{array}{c}\text { Grandes } \\
\text { sistemas }\end{array}$ & $\begin{array}{c}\text { Análisis } \\
\text { en línea }\end{array}$ & $\begin{array}{c}\text { Mayor } \\
\text { Flexibilidad }\end{array}$ & $\begin{array}{c}\text { Menor } \\
\text { Tiempo } \\
\text { ejecución }\end{array}$ & $\begin{array}{c}\text { Precisión } \\
\text { eventos } \\
\text { reales }\end{array}$ & $\begin{array}{c}\text { Menor } \\
\text { limitación }\end{array}$ & $\begin{array}{c}\text { Menores } \\
\text { Costos }\end{array}$ \\
\hline Flujos convencionales & 9 & 8 & 5 & 8 & 7 & 10 & 10 \\
\hline Flujo progresivos & 8 & 6 & 5 & 7 & 7 & 8 & 9 \\
\hline Análisis Dinámicos & 7 & 5 & 7 & 6 & 9 & 7 & 8 \\
\hline Monitoreo & 6 & 10 & 10 & 9 & 9 & 3 & 2 \\
\hline
\end{tabular}

En la Tabla 1, se observan que las ventajas de los métodos de análisis estáticos basados en flujos convencionales son su menor costo de implementación, la rapidez de ejecución, las menores limitaciones para la implementación y que pueden ser útiles para el análisis en grandes sistemas de potencia; esto se debe a que se basan en ecuaciones simples que representan bien la estabilidad de voltaje y pueden ser resueltos sin muchos recursos computacionales (Pal, 1992; Kundur, 1994; Chowdhury et al., 2000). Entre las desventajas de estos métodos se encuentran que no tienen suficiente flexibilidad ante los cambios del sistema y deben actualizarse constantemente, tampoco tienen tanta precisión a eventos reales como los dinámicos o monitoreo debido a los modelos de elementos que utilizan (Morison et al., 1993; Chowdhury et al., 2000; Huadong et al., 2005; Muhammad Nizam, 2006). Los métodos estáticos basados en flujos progresivos ofrecen mayor precisión en los cálculos cercanos al límite de estabilidad de voltaje que los métodos basados en 
flujos convencionales (Ajjarapu et al., 1991, Kundur, 1994, Zarate et al., 2006), debido a su algoritmo de búsqueda de la solución; son métodos que pueden trabajarse en grandes sistemas de potencia (Cañizares, 1993), sus costos de implementación son muy bajos. Entre las desventajas de estos métodos se encuentran que no son flexibles a los cambios y no ofrecen tanta precisión a eventos reales como los métodos dinámicos por sus modelos de elementos; además los tiempos de ejecución son mayores que los métodos basados en flujos convencionales (De Souza et al., 2000; Zarate et al., 2006). Las métodos dinámicos cuentan con mayor precisión a eventos reales, sus costos de implementación son bajos comparados a los de monitoreo, son más flexibles que los estáticos y funcionan bien para grandes sistemas de potencia; entre sus desventajas se encuentran los requerimientos de mayores recursos computacionales y las simulaciones requieren de mayor tiempo de ejecución que para los métodos estáticos debido a las ecuaciones algebraicas que deben resolverse en el tiempo (Morison et al., 1993; Huadong et al., 2005), no calculan la estabilidad de voltaje directamente y algunas aplicaciones de análisis dinámico que utilizan ecuaciones simplificadas del estado transitorio, pierden precisión a los eventos reales (Van Cutsem y Vournas, 1996; Chowdhury et al., 2000). Los métodos de monitoreo cuentan con la ventaja que se pueden utilizar en línea o fuera de línea con datos reales del sistema, lo cual permite representar eventos con mayor precisión, pero requieren en muchos casos, combinación con métodos estáticos y dinámicos para calcular los límites y márgenes del sistema. La utilización de algoritmos expertos que identifiquen las condiciones del sistema ayudan a mejorar los tiempos de ejecución (Chakrabarti y Jeyasurya, 2004; Kamalasadan et al., 2006); pueden tener desventajas dependiendo de la técnica de análisis utilizada, la cantidad de datos, la calidad de los datos; requieren de representación matemática exacta, equipos de comunicación, computación y software especializado, lo cual aumenta los costos de implementación y hace que para grandes sistemas su tiempo de ejecución sea aumentado y tengan mayor dificultad en la aplicación (Chakrabarti y Jeyasurya, 2004).

\section{CONCLUSIONES}

En este documento se presentó una clasificación general de los métodos utilizados para el estudio de la estabilidad de voltaje, basados en la predicción y la detección del problema. Se hace referencia a los desarrollos del tema en orden cronológico, sus modificaciones y utilización en la solución de la estabilidad de voltaje. Una comparación de cada método muestra las ventajas y desventajas, lo cual puede utilizarse como guía para la implementación y el desarrollo de nuevas técnicas enfocadas a mejorar la estabilidad de voltaje, de acuerdo a las características del método de prevención y corrección a utilizar en la solución. Los factores importantes que pueden destacarse de las comparaciones de los método son: los menores tiempos de ejecución y menores limitaciones en la implementación de los análisis estáticos, la precisión de los métodos dinámicos y monitoreo, los bajos costos de implementación de los métodos estáticos y dinámicos, la flexibilidad y aplicabilidad en línea de los métodos de monitoreo.

\section{AGRADECIMIENTOS}

A COLCIENCIAS y al Grupo de investigación en Alta Tensión (GRALTA) de la Universidad del Valle, Cali, Colombia por su colaboración económica.

\section{REFERENCIAS}

Aik, D.L.H. y G. Andersson; Voltage Stability Analysis of Multi-Infeed HVDC Systems, IEEE Transactions on Power Delivery: 12(3), 1309-1318 (1997).

Aik, D.L.H. y G. Andersson; Use of participation factors in modal voltage stability analysis of multi-infeed HVDC systems, IEEE Transactions on Power Delivery; 13(1), 203-211 (1998).

Ajjarapu, V. y B. Lee; Bibliography on voltage stability, IEEE Transaction on Power Systems: 13(1), 115-125, (1998).

Ajjarapu, V. y C. Christy; The application of a locally parameterized continuation technique to the study of 
steady state voltage stability, Proceedings of the Twenty-First Annual North-American: 161-167 (1989).

Ajjarapu, V. y C. Christy; The continuation power flow: a tool for steady state voltage stability analysis, Conference Proceedings, Power Industry Computer Application Conference: 304-311 (1991).

Amjady, N. y M. Esmaili; Application of a new sensitivity analysis framework for voltage contingency ranking, IEEE Transaction on Power Systems: 20(2), 973-983 (2005).

An Ning, Zhou Shuangxi y Zhu Lingzhi; Power System Voltage Stability Limits Estimation based on Quasi-Steady-State Simulation, International Conference on Power System Technology: 1-7, (2006).

Aumuller, C. y T.K. Saha; Analysis and assessment of large scale power system voltage stability by a novel sensitivity based method, IEEE Power Eng. Society Summer Meeting: 3, 1621-1626 (2002).

Bedoya, D.B. y C.A. Castro; Computation of Power Systems Minimum Voltage Stability Security Margins, International Conference on Power System Technology: 1-7 (2006).

Begovic, M.M. y A.G. Phadke; Control of voltage stability using sensitivity analysis, IEEE Transactions on Power Systems: 7(1), 114-123 (1992).

Belhadj, C.A., H- Al- Duwaish, M.H. Shwehdi y A.S. Farag; Voltage stability estimation and prediction using neural network, International Conference on Power System Technology: 8(2), 1464-1467 (1998).

Berizzi, A. y otros cuatro autores; Singular value decomposition for an ORPF formulation in presence of SVR, IEEE Mediterranean Electrotechnical Conference: 968- 972 (2006).

Berntsen, T.O., N. Flatabo, J.A. Foosnaes y A. Johannesen; Sensitivity signals in detection of network condition and planning of control actions in a power system, CIGRE-lb AC Symposium on Control Applications for Power System Security: 208(03) (1983).

Bucciero, J. y M. Terbrueggen; Interconnected Power System Dynamics Tutorial: Dynamics of Interconnected Power Systems Tutorial, Electric Power Reasearch Institute, EPRI, Ed: 3, 6.1-6.55 (1998).

Cañizares, C.A.; Voltage collapse and transient energy function analysis of ac/dc systems, PhD thesis, University of Wisconsin-Madison (1991).

Cañizares, C.A. y F.L. Alvarado; Point of collapse and continuation methods for large AC/DC systems, IEEE Transaction Power Systems: 7(1), 1-8 (1993).

Cañizares, C.A., A.C.Z. De Souza y V.H. Quintana; Comparison of performance indices for detection of proximity to voltage collapse, IEEE Transactions on Power Systems: 11(3), 1441-1450 (1996).

Capitanescu, F. y T. Van Cutsem; Unified sensitivity analysis of unstable or low voltages caused by load increases or contingencies, IEEE Transactions on Power Systems: 20(1), 321-329, (2005).

Chakrabarti, S. y B. Jeyasurya; On-line voltage stability monitoring using artificial neural network, Large Engineering systems Conference on Power Engineering (2004).

Chebbo, A.M., M.R. Irving y M.J.H. Sterling; Voltage collapse proximity indicator: behavior and implications, IEEE Proceedings-Generation, Transmission and Distribution: 139(3), 241-252 (1992).

Chih-Wen, Liu, Chen-Sung Chang y Mu-Chun Su; Neuro-Fuzzy networks for voltage security monitoring based on synchronized phasor measurements, IEEE Transactions on Power Systems: 13(2), 326-332 (1998). 
Chowdhury, B.H. y C.W. Taylor; Voltage stability analysis: V-Q power flow simulation versus dynamic simulation, IEEE Transactions on Power Systems: 15(4), 1354-1359 (2000).

DeMarco C.L. y T.J. Overbye; An energy-based measure for assessing vulnerability to voltage collapse, IEEE Transaction on Power Systems:, PWRS-5, 419-427 (1990).

De Souza, A.C.Z., C.A. Cañizares y V.H. Quintana; New techniques to speed up voltage collapse computations using tangent vectors, IEEE Transactions on Power Systems: 12(3), 1380-1387 (1997).

De Souza, A.C.Z., J.C.S. de Souza y A.M.L. da Silva; On-line voltage stability monitoring, IEEE Transactions on Power Systems: 15(4), 1300-1305 (2000).

El-Sheikhi, S., S. Osman y A. El-Arroudi, Voltage Stability assessment using modal analysis of power systems including Flexible AC Transmission System (FACTS), Large Engineering Systems Conference on Power Engineering: 105-108, (2003).

Esaka, T., Y. Kataoka, T. Ohtaka y S. Iwamoto: Voltage stability preventive and emergency preventive control using VIPIt sensitivities, IEEE PES Power Systems Conference and Exposition: 509-516 (2004).

Feng, Z., V. Ajjarapu y D.J. Maratukulam; A practical minimum load shedding strategy to mitigate voltage collapse, IEEE Transactions on Power Systems: 13(4), 1285-1291 (1998).

Flatabo, N., R. Ognedal y T. Carlsen; Voltage stability condition in a power transmission system calculated by sensitivity methods, IEEE Transaction on Power Systems: 5(4), 1286-1293 (1990).

Gao, B., G.K. Morison y P. Kundur; Voltage stability evaluation using modal analysis, IEEE Transaction on Power Systems: 7(4), 1529-1542 (1992).

Garng H. y H. Zhang; Dynamic voltage stability reserve studies for deregulated environment, IEEE Power Engineering Society Summer Meeting: 1, 301-306 (2001).

Gelopulos, D.P.; Midterm Simulation of Electric Power Systems, EPRI 745 -596. (1979).

Grigsby, L.L.; The electric power engineering handbook, Ed. Boca Raton: CRC Press LLC (2001).

Hae-Kon, N., K. Yong-Ku, S. Kwan-Shik y K.Y. Lee; A new eigen-sensitivity theory of augmented matrix and its applications to power system stability analysis. IEEE Transactions on Power Systems: 15(1), 363369 (2000).

Halim, A., K. Takahashi y B. Kermanshahi; Lyapunov function for robust voltage stability analysis considering perturbation location, Proceedings, International Conference on Power System Technology: 2, 1508-1512 (1998a).

Halim, A., K. Takahashi y B. Kermanshahi; Dynamical voltage stability analysis using Lyapunov function method, Proceedings. 8th International Conference on Harmonics and Quality of Power: 1, 439-444 (1998b).

Haque, M.H.; Determination of steady-state voltage stability limit using $P$-Q curve, IEEE Power Engineering Review: 22(4), 71-72 (2002).

Haque, M.H.; Novel method of assessing voltage stability of a power system using stability boundary in $P-Q$ plane, Electric Power System Research: 64(1), 35-40 (2003a).

Haque, M.H.; On-line monitoring of maximum permissible loading of a power system within the voltage stability limits, IEE Proceeding-Generation, Transmission, Distribution: 150(1), 107-112 (2003b).

Haque, M.H.; Use of V-I characteristic as a tool to assess the static voltage stability limit of a power 
system, IEE Proceedings, Generation, Transmission and Distribution: 151(1), 1-7 (2004).

Hawkins, N.T., G. Shackshaft y M.J. Short; Online algorithms for the avoidance of voltage collapse: reactive power management and voltage collapse margin assessment, Third International Conference on Power System Monitoring and Control, London, UK, 134-139 (1991).

Hsiao-Dong Chiang, Cheng-Shan Wang y Alexander J. Flueck; Look-ahead voltage and load margin contingency selection functions for large-scale power systems IEEE Transactions on Power Systems:12(1), 173-180 (1997).

Huang, Z., L. Bao y W. Xu; Generator ranking using modal analysis, IEE Proceedings, Generation, Transmission and Distribution: 150(6), 709-716 (2003).

Huadong Sun, Xiaoxin Zhou y Ruomei Li; Accuracy Analysis of Static Voltage Stability Indices Based On Power Flow Model, 2005 IEEE/PES Transmission and Distribution Conference and Exhibition: Asia and Pacific: 1-7 (2005).

IEEE/PES Power System Stability Subcommittee, Voltage Stability Assessment: Concepts, Practices and Tools, special publication, final draft, (2003).

Jasmon, G.B. y L.H.C.C. Lee; New contingency ranking technique incorporating a voltage stability criterion, IEE Proceedings-Generation, Transmission and Distribution: 140(2), 87-90 (1993).

JiaKuan X. y M. Xin, Bifurcation analysis for power system voltage stability based on singular perturbation method, Intern. Conf. on Electrical Machines and Systems: 1811-1814 (2007).

Julian, D.E. y otros cinco autores; Quantifying proximity to voltage collapse using the voltage instability predictor (VIP), Proceeding IEEE PES Summer Meeting, Seattle WA: 2, 931-936 (2000).

Kamalasadan, S., A.K. Srivastava y D. Thukaram; Novel algorithm for online voltage stability assessment based on feed forward neural network, IEEE Power Engineering Society General Meeting: 1-7 (2006).

Kataoka, Y., M. Watanabe y S. Iwamoto; A New Voltage Stability Index Considering Voltage Limits, IEEE PES Power Systems Conference and Exposition: 1878-1883 (2006).

Koessler, R.J., W. Qiu, M. Patel y H.K. Clark; Voltage Stability Study of the PJM System Following Extreme Disturbances, IEEE Transactions on Power Systems: 22(1), 285-293 (2007).

Kundur, P.; Power system stability and control, Ed. McGraw-Hill, 17-39, 959-1020 (1994).

Kundur, P. y otros 11 autores; Definition and classification of power system stability. IEEE transactions on power systems: 19(2), 1387-1401 (2004).

Limbu, T.R., T.K. Saha y J.D.F. McDonald; Comparing Effectiveness of Different Reliability Indices in Contingency Ranking and Indicating Voltage Stability. Universities Power Engineering Conference (2005).

Lixin Bao, Zhenyu Huang y Wilsun Xu; On-line voltage stability monitoring using VAr reserves, IEEE Power Engineering Society General Meeting: 3, 1754-1754 (2003).

Lof, P.-A., G. Andersson y D.J. Hill; Voltage stability indices for stressed power systems, IEEE Transactions on Power Systems: 8(1), 326-335 (1993).

Lof, P.-A., T. Smed, G. Andersson y D.J. Hill; Fast calculation of a voltage stability index, IEEE Transactions on Power Systems: 7(1), 54-64 (1992). 
Lopez-Luis, E., M.A. Garcia-Dominguez y D. Ruiz-Vega: The effect of improved system modeling in the continuation power flow method, Bulk Power System Dynamics and Control-VII. Revitalizing Operational Reliability, IREP Symposium (2007).

Mili, L., T. Baldwin y R. Adapa; Phasor measurement placement for voltage stability analysis of power systems, Proceedings of the 29th IEEE Conference on Decision and Control: 6, 3033-3038 (1990).

Mori, H. y Y. Tamaru; A decentralized scheme for voltage instability monitoring with hybrid artificial neural networks, Proceedings of the Second International Forum on Applications of Neural Networks to Power Systems: 378-381 (1993).

Mori, H. y T. Kojima; Hybrid continuation power flow with linear-nonlinear predictor, International Conference on Power System Technology, POWERCON 2004: 1, 969-974 (2004).

Morison, G.K., B. Gao y P. Kundur; Voltage stability analysis using static and dynamic approaches, IEEE Transactions on Power Systems: 8(3), 1159-1171 (1993).

Nagao, T., K. Tanaka y K. Takenaka;, Development of static and simulation programs for voltage stability studies of bulk power system, IEEE Transactions on Power Systems: 12(1), 273-281 (1997).

Nino, E.E., C.A. Castro, L.C.P. da Silva y A.A. Alves; Continuation load flow using automatically determined branch megawatt losses as parameters, IEE Proceedings Generation, Transmission and Distribution: 153(3), 300-308 (2006).

Muhammad Nizam, Azah Mohamed y Aini Hussain; Performance Evaluation of Voltage Stability Indices for Dynamic Voltage Collapse Prediction, Journal of Applied Sciences: 6(5), 1104-1113 (2006).

Overbye, T.J.; Use of Energy Methods for On-Line Assessment of Power System Voltage Security, IEEE Trans. on Power Systems: 8(2), 452-458 (1993).

Pal M.K.; Voltage stability conditions considering load characteristics, IEEE transactions on power systems: 7(1), 243-249 (1992).

Parniani, M., J.H. Chow, L. Vanfretti y B. Bhargava; A. Voltage Stability Analysis of a Multiple-Infeed Load Center Using Phasor Measurement Data, IEEE PES Power Systems Conference and Exposition: 1299-1305 (2006).

Reis, C. y F.P.M. Barbosa; A comparison of voltage stability indices, IEEE Mediterranean Electrotechnical Conference: 1007-1010 (2006).

Sami Repo; On-line voltage stability assessment of power system-An approach of black-box modeling, Tampere University of Technology: (344) (2001).

Sanaye-Pasand, M. y A. Rezaei-Zare; Review and Simulation of Static Indices in Detection of Proximity to Voltage Collapse, Australian Universities Power Engineering Conference, (2003).

Santos, J.L. y F.P.M. Barbosa; Voltage Stability Evaluation Using the Relation between Impedances, IEEE MELECON 2004, Croatia: 927-930 (2004).

Schmidt, H. P.; Application of artificial neural networks to the dynamic analysis of the voltage stability problem, IEE Proceeding-Generation, Transmission, Distribution: 4(4), 371-377 (1997).

Schulz, R. P. y A.E. Turner; Long-Term Power System Dynamics, EPRI Research Project 764-2, EL-983 (1980).

Sharma, N.K., A. Ghosh y R.K. Varma; A novel placement strategy for FACTS controllers, IEEE Transactions on Power Delivery: 18(3), 982-987 (2003). 
Sharma, C. y G.M. Ganness; Determination of Power System Voltage Stability Using Modal Analysis, Intern. Conf. on Power Eng., Energy and Electrical Drives: 381-387 (2007).

Sinha A.K. y D. Hazarika; A comparative study of voltage stability indices in a power system, Electrical Power and Energy Systems: 22, 589-596 (2000).

Sobierajski, M. y M. Fulczyk; Use of p-q curve with rectangular probability distribution of bus load in voltage stability study, IEEE PES Power Systems Conference and Exposition: 1, 130-136 (2004).

Sobierajski, M., K. Wilkosz, M. Fulczyk y J. Bertsch; Using Bus Impedance and Bus P-Q Curve for Voltage Stability Control, IEEE/PES Transm. and Distr. Conf. and Exposition: 1, 79-84 (2001).

Sode-Yome, A., N. Mithulananthan y K.Y. Lee; A maximum loading margin method for static voltage stability in power systems, IEEE Transactions on Power Systems: 21(2), 799-808 (2006).

Soliman, S.A., H.K. Temraz y S.M. El-Khodary; Power System Voltage Stability Margin identification Using Local Measurements, IEEE Large Engineering Systems Conference on Power Engineering: 100104 (2003).

Song, H. y otros cuatro autores, Determination of interface flow margin using the modified continuation power flow in voltage stability analysis, IEE Proceeding-Generation, Transmission, Distribution: 148(2), 128-132 (2001).

Su, Y.C., S.J. Cheng, J.Y. Wen y J. Zhang; Power System Dynamic Stability Analysis and Stability Type Discrimination, Proc. of the 41st International Universities Power Engineering Conference: 1-6 (2006).

Tamura, Y., K. Sakamoto y Y. Tayama; Voltage instability proximity index (VIPI) based on multiple load flow solutions in ill-conditioned power systems, Proceeding of the 27th IEEE Conference on Decision and Control (1988).

Taylor, C.W.; Power System Voltage Stability, Ed. McGraw-Hill (1994).

Thomas y H-D. Chiang; On the Dynamics of Voltage Instabilities, Proceedings Bulk Power System Voltage Phenomena- Voltage Stability and Security, 6.1-6.21 (1989).

Thukaram D. y otros cuatro autores; Voltage stability improvement: case studies of Indian power networks, Electric Power Systems Research: 44(1), 35-44, (1998).

Uhlen, K., L. Warland J.O. Gjerde, K. VU Ø. Kirkeluten; Concepts for intelligent monitoring and control of power grids by use of new measurement technologies, CIGRE (2004).

Vaahedi, E. y otros cinco autores; Voltage stability contingency screening and ranking, IEEE Transactions on Power Systems: 14(1), 256-265 (1999).

Vaahedi, E., J. Tamby, Y. Mansour y D. Wenyuan Li Sun; Large scale voltage stability constrained optimal VAr planning and voltage stability applications using existing OPF/optimal VAr planning tools, IEEE Transactions on Power Systems: 14(1), 65-74 (1999).

Van Cutsem T., Y. Jacquemart, J.-N. Marquet y P. Pruvot; Extensions and applications of a mid-term voltage stability analysis method, Proc. 3rd Intern. Workshop on Bulk Power System Voltage Phenomena - Voltage Stability and Security: 251-270 (1994).

Van Cutsem, T., Y. Jacquemart, J.N. Marquet y P. Pruvot; A comprehensive analysis of mid-term voltage stability, IEEE Transactions on Power Systems: 10(3), 1173-1182 (1995). 
Van Cutsem T y C.D. Vournas; Voltage stability analysis in transient and mid-term time scales, IEEE Transactions on Power Systems: 11(1), 146-154 (1996).

Van Cutsem T. y R. Mailhot; Validation of a fast voltage stability analysis method on the HydroQuebec system, IEEE Transactions on Power Systems: 12(1), 282-292 (1997).

Venikov, V.A., V.A. Stroev, V.I. Idelchick y V.I. Tarasov; Estimation of Electrical Power System SteadyState Stability in Load flow Calculations, IEEE Transaction on Power Apparatus and Systems: 94, (3), 1034-1041 (1975).

Vournas, C.D.; Voltage stability and controllability indices for multimachine power systems, IEEE Transactions on Power Systems: 10(3), 1183-1194, (1995).

Vu, K., M.M. Begovic, D. Novosel y M.M. Saha; Use of local measurements to estimate voltage-stability margin, IEEE Transaction On Power Systems: 14(3), 1029-1035 (1999).

Wang Q., H. Song y V. Ajjarapu; Continuation-based quasi-steady-state analysis, IEEE Transactions on Power Systems: 21(1), 171-179 (2006).

Wiszniewski, A.; New Criteria of Voltage Stability Margin for the Purpose of Load Shedding, IEEE Transactions on Power Delivery: 22(3) (2007).

Xu, W. e Y. Mansour; Voltage Stability Analysis Using Generic Dynamic Load Models, IEEE Transactions on Power Systems: 9(1), 479-493 (1994).

Yang, Z, P. Cao y M.L. Crow; A New Computationally Efficient Index for loadability limit studies Electric Machines and Power Systems, Taylor \& Francis, 28, 385-397 (2000).

Yihong Wang, Chen Shen, Shengwei Mei y Ancheng Xue; Analysis of Transient Voltage Stability via Quadratic Approximation Method, Conference on International Power System Technology: 1-6 (2006).

Yorino, N. y otros cuatro autores; A new continuation power flow based on Q-limit points, The 2004 47th Midwest Symposium on Circuits and Systems: 2, II-341 - II-344, (2004).

Zarate, L.A.L. y C.A. Castro; Fast computation of security margins to voltage collapse based on sensitivity analysis, IEE Proceedings Generation, Transmission and Distribution: 153(1), 35-43 (2006).

Zarate, L.A.LI., C.A. Castro, J.L.M. Ramos y E.R. Ramos; Fast computation of voltage stability security margins using nonlinear programming techniques, IEEE Transactions on Power Systems: 21(1), 19-27, (2006).

Zhang, X.-P., P. Ju y E. Handschin; Continuation Three-Phase Power Flow: A Tool for Voltage Stability Analysis of Unbalanced Three-Phase Power Systems, IEEE Transactions on Power Systems: 20(3), 1320-1329 (2005).

Zhao J. y otros cuatro autores; On-Line Voltage Stability Monitoring and Control (VSMC) System in Fujian power grid, IEEE Power Engineering Society General Meeting, 1-6 (2007). 